\title{
Wireless Manipulation of Single Cells using Magnetic Microtransporters
}

\author{
Mahmut Selman Sakar ${ }^{1,2}$, Edward B. Steager ${ }^{1}$, Anthony Cowley ${ }^{1}$, Vijay Kumar ${ }^{1}$, and George J. Pappas ${ }^{1}$ \\ ${ }^{1}$ GRASP Laboratory, University of Pennsylvania, Philadelphia, PA 19104 \\ ${ }^{2}$ Department of Mechanical Engineering, Massachusetts Institute of Technology, Cambridge, MA 02139
}

\section{ABSTRACT}

For such biomedical applications as single cell manipulation and targeted delivery of chemicals, it is important to fabricate microstructures that can be powered and controlled without a tether in fluidic environments. In this work, we describe the construction and operation of micronsized, biocompatible ferromagnetic microtransporters driven by external magnetic fields capable of exerting forces at the pico Newton scale. We develop microtransporters using a simple, single step micro fabrication technique that allows us to produce large numbers in the same step. We also fabricate microgels to deliver drugs. We demonstrate that the microtransporters can be navigated to separate individual targeted cells with micron-scale precision, and deliver microgels without disturbing the cells in the neighborhood and the local microenvironment.

Keywords: magnetic actuation, microrobot, single cell manipulation, microgels, in vitro.

\section{INTRODUCTION}

As the length scales of robotic systems continue to decrease, one of the clear emerging applications is the manipulation of single biological cells in fluid environments. Single-cell manipulation has traditionally been achieved with pipettes, optical tweezers, or specialized microfluidic channel designs [1]. Recently, a variety of techniques have been explored for the wireless control of microrobots. While some of these methods directly integrate motile microorganisms into the design [2]-[5], other bio-inspired methods rely on controlling applied magnetic forces [6], [7]. Magnetic control of microrobots and microgrippers has also been established as an effective means of microobject manipulation [8][11]. However, significant challenges remain for applications relating to single cell manipulation mainly due to appropriate scaling of robot size and geometry of existing designs.

One of the most important length scales to consider for the system is the workspace for the robot. When working with single cells, fine details of individual cells must be

${ }^{*}$ We would like to thank Dr. Sean Kim for his help in culturing rat hippocampal neurons, Prof. James Eberwine and Prof. Junhyong Kim for identifying the problem of single cell manipulation and Prof. C. J. Taylor for fruitful discussions. We gratefully acknowledge the support of ARO MURI Grant W911NF-05-1-0219 and Penn Genome Frontier Institute.

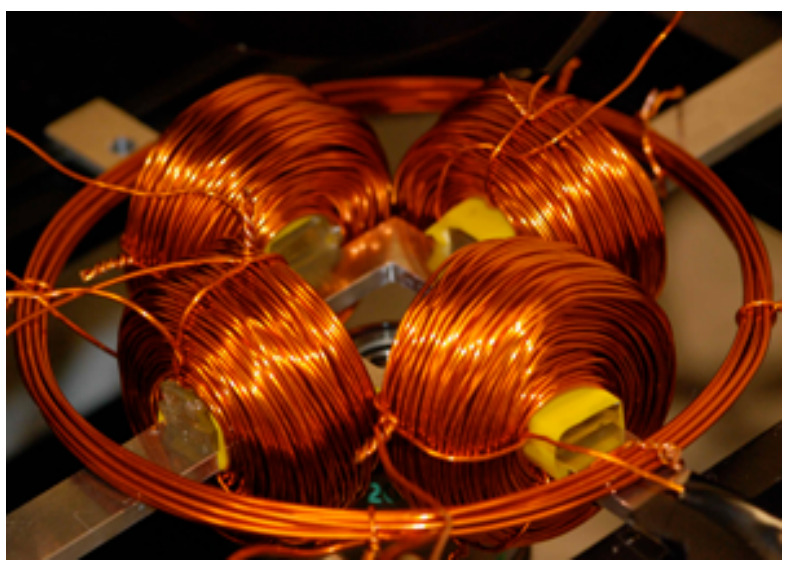

Fig. 1. Five electromagnetic coils, four in-plane and one out-of-plane, are mounted on an optical microscope to actuate the microtransporters.

resolved. It is essential to have microstructures with sizes comparable to those of target cells in order to transport and position them with precision. The mammalian cell is an entity with typical dimensions of tens of microns. This requires a magnification of at least $40 \mathrm{X}$. The workspace is then $150 \mu \mathrm{m} \times 150 \mu \mathrm{m}$. Based on this, it becomes clear that the robot must not only be small relative to the workspace, but also that precise control of movement is very important. In fact, rapid movements may cause significant disturbances to the microenvironment.

Robotic manipulators on the scale of cells offer significant benefits beyond simply moving cells. Wirelessly controlled (i.e. untethered) cell-sized robots are highly non-invasive. At this length scale, where viscous fluid forces dominate inertial forces, motile microrobots cause very little mixing or agitation of the surrounding environment. This is a significant advantage over suction pipetting for life scientists, since pipettes cause relatively large fluid disturbances. Traditionally, the focus of robotic manipulators has been centered on applying mechanical forces. However, on the scale of individual cells, the understanding of the word manipulation itself must be expanded to include chemical manipulation of local microenvironments. To a great extent, research in single cell life sciences is concerned with biochemistry. Due to this, a system for the delivery of chemicals in the microenvironment would greatly enhance the usefulness of 
a microrobotic system.

The microtransporter, which is only slightly larger than the rat hippocampal neurons which we are interested in manipulating, has been designed to work on a scale appropriate for the working space of a standard optical microscope. It is aligned by magnetic fields and pulled by field gradients. An oscillating out-of-plane magnetic field induces a stick/slip mechanism that enhances control of the robot [12]. This is useful not only for adjusting the velocity of the robot [9], but also for traversing irregular microscale topographies such as surfaces densely patterned with adherent cells. Composed of iron oxide nanoparticles embedded in a polymer, the robot is fully biocompatible and is patterned using a single-mask photolithographic process. The robot is similar in density to the working fluid. Thus, very small magnetic forces are required for movement. Furthermore, due to the sub-micron resolution of the photolithographic micromachining process, the robot's shape can be tailored to and scaled appropriately for geometric compatibility with different cell types. Release in the microenvironment is enabled by a biocompatible, water-soluble etch process. Visual servoing is used for both teleoperated and fully automated manipulation. We demonstrate this here using latex microbeads and rat hippocampal neurons. Finally, we present results on the integration of microscale hydrogels designed for the localized delivery of chemicals using the microrobot.

\section{EXPERIMENTAL SETUP AND FABRICATION OF MAGNETIC MICROTRANSPORTERS}

The experimental setup consists of four identical in-plane electromagnetic coils with diameter $3.5 \mathrm{~cm}$ placed $3.5 \mathrm{~cm}$ away from each other and one out-of-plane electromagnetic coil with diameter $8 \mathrm{~cm}$ (See Figure 1). The coils are integrated with an aluminum frame that allows experimentation with both inverted and upright microscopes. The coils are independently driven with $\mathrm{H}$-bridge motor drivers and current control electronics. Imaging is performed on a Nikon inverted microscope using phase contrast. Videos are captured using a CCD camera. Fluorescent images of microgels were taken under a Zeiss microscope supported with a $100 \mathrm{~W}$ mercury lamp and filter sets.

In our previous work, we developed a single step fabrication process for biocompatible magnetic microtransporters that did not require subsequent lithography or etching processes [9]. In this work, we build on our previous results while making several improvements. First, we reduce the size of our transporters one order of magnitude. Second, we increase throughput of the microfabrication process. Third, our process yields robots that are free of the excess iron oxide particles that can be seen in the micrographs in [9]. The first spin-coating procedure is used to prepare the nontoxic water-soluble sacrificial dextran layer [13]. We need this layer to release microstructures into the fluidic chamber without causing any structural damage. Compared to our

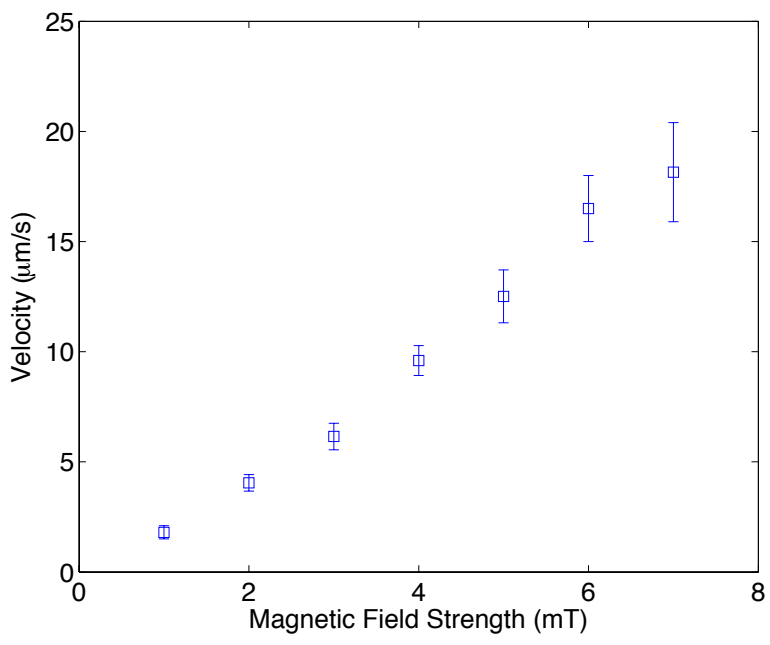

Fig. 2. Microtransporter velocity as a function of magnetic field strength. Error bars indicate one standard deviation error.

previous protocol, we increase the concentration of dextran $50-70 \mathrm{kDa}$ from $5 \%(\mathrm{w} / \mathrm{v})$ to $10 \%(\mathrm{w} / \mathrm{v})$ and decrease the spin coating speed from $2000 \mathrm{rpm}$ to $1000 \mathrm{rpm}$ to obtain a thicker and more resistant sacrificial layer. Next, a thin layer $(2 \mu m)$ of pure SU8-2 is spin coated. This extra layer ensures better release of microtransporters and helps to obtain a more uniform coating of composite polymer in the following step. Finally, the composite ferromagnetic photoresist is spin coated and the exposed substrate is postbaked and developed. We optimize our fabrication procedure for a specific weight ratio (5\% by weight) and photoresist thickness $(10 \mu \mathrm{m})$ and fabricate $30 \times 30 \times 10 \mu \mathrm{m}^{3} \mathrm{U}-$ shaped microtransporters.

We magnetize our microtransporters using a rectangular neodymium-iron-boron $(\mathrm{NdFeB})$ magnet with a surface field of 6450 Gauss (K\&J Magnetics, Jamison, PA) in the direction of the opening of the $U$ shape so that the magnetization vector points towards that direction. They are released on a glass slide by bringing the chip with patterned microstructures into contact with DI water. They can also be trapped and released inside a microfluidic channel for microfluidic applications [14].

\section{Motion Control And Visual Tracking}

\section{A. Motion Control}

The robot motion is a stick-slip motion similar to the phenomenon reported in [15]. By applying a time-varying magnetic field using electromagnetic coils we can control the stick-slip motion. An in-plane field is applied to orient the microtransporters but the force exerted is not high enough to overcome the frictional forces to translate them. A sinusoidal out-of-plane field with an amplitude of $1 \mathrm{mT}$ is applied using the electromagnetic coil placed above the surface which 
induces a rocking motion. The main difference with [15] lies in the scale of the robots and their magnetization. In contrast to this previous work which relies on hard magnets, our robots are based on ferromagnetic particles which are magnetized as needed. More importantly, our robots are at the same scale as the target mammalian cells.

In our own previous work, we characterized the velocity of the transporters with respect to the pulsing frequency and showed that the velocity increases monotonically with increasing frequency [9]. In this work, we fix the pulsing frequency at $30 \mathrm{~Hz}$ and adjust the in-plane magnetic field strength to control the velocity of the transporters. We performed several trials with different microrobots by varying the current passing through the in-plane coils. Velocity increases linearly with increasing magnetic field strength (see Figure 2). If we assume that the robots have uniform magnetization $M$, the magnetic force exerted on the robots is given by,

$$
F=V(M \cdot \nabla) B
$$

where $V$ is the volume of the microtransporter and $B$ is the applied magnetic field. As the Reynolds number is relatively low, viscous forces dominate inertial effects and velocity of the robot is directly proportional to the applied force. Combined with (1), a linear trend between velocity and magnetic field strength is expected. Furthermore, the small values of the velocity can be explained by the size of the robots as exerted force is a function of size.

\section{B. Visual Tracking}

The visual tracking system fuses several individually unreliable detectors and estimators to establish a stable estimate of manipulator pose. In order to alleviate the burden on experimental procedure, very few constraints are placed on expected image backgrounds or absolute image characteristics. Instead, relative measures are preferred, and processing is broken into a series of stages that iteratively refine the region of interest. The output of the entire tracking scheme running at $30 \mathrm{~Hz}$ is highlighted in Figure 3.

The first winnowing of the field of view is a block matching optical flow estimation that filters out small, unfocused particles. The centroid of the largest region of moving blocks, shown as a blue rectangle, is used to identify the center of interest for subsequent processing.

The image of the manipulator is primarily characterized by a dark outline, a light interior, and a cup-like $(\sqcup)$ shape. None of these features can be modeled too rigidly however, as the outlines of the manipulators used in different experiments will be corrupted by particles in the environment adhering to the manipulator itself, while the lighter interior of the image is often broken up by particles sticking to the top of the manipulator. Rather than attempt to extract a closed contour for the manipulator's perimeter, we find an oriented rectangular structure by considering projections of dark pixels.

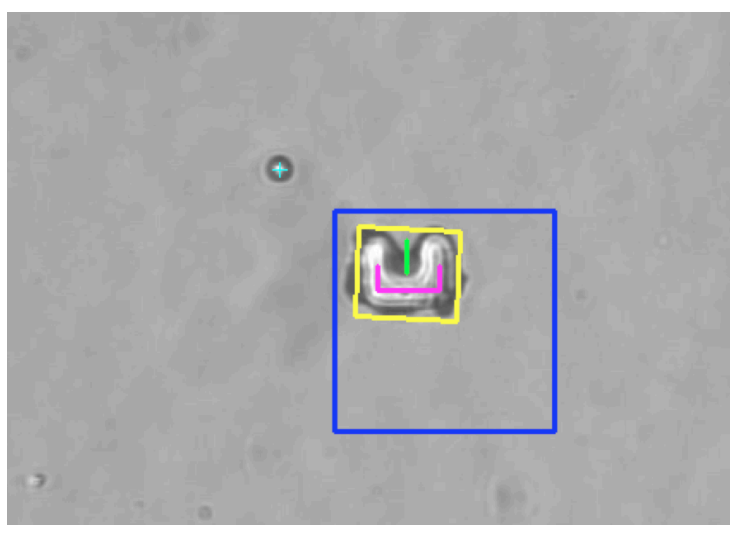

Fig. 3. Micromanipulator visual tracking output. The tracker estimates the position and orientation of the manipulator in $2 \mathrm{D}$, as well as the positions of polystyrene beads.

Locally dark pixels are projected into a histogram associated with each orientation, and the pair of histograms associated with orthogonal projections with the lowest entropy bimodal distributions along the axes of projection are used to identify an orientation, localization, and scale of the likely outline of the manipulator. The resulting rectangle is shown in yellow in Figure 3.

This rectangle is used to guide a procedure that fits a model of the manipulator's light interior region. A skeleton of the connected component comprising the brightest pixels inside the estimated perimeter is fed into a shape detector that searches for a minimal entropy bimodal projection perpendicular to a unimodal projection. These projections, shown in magenta, correspond to the parallel uprights and the base of the idealized cup shape. The manipulator can rotate instantaneously, but tends to translate at a more measured pace. A motion model consistent with this observation is used to guide a particle filter on the structure's pose, shown by the green line segment in the figure.

Finally, beads are tracked by feeding the output of a Hough transform-based circle detector into a particle filter. An example bead detection is show by a cyan + symbol in Figure 3.

\section{FABRICATION OF MICROGELS}

SU8 master posts are fabricated on a silicon wafer using photolithography. We form poly(dimethyl siloxane) (PDMS) molds to serve as templates for microgel structures using replica molding. To prepare the PDMS molds for treatment with the gel, we oxidize them in air plasma to render their surfaces hydrophilic. They can also be placed in jars filled with water and degassed under house vacuum to remove air bubbles as suggested in [16] to increase throughput. Agarose gel is prepared by mixing 6\% agarose with DI water and heating the mixture in a microwave oven until the agar is completely dissolved. Molten solution is immediately poured onto the PDMS mold while it is hot and excess solution is 


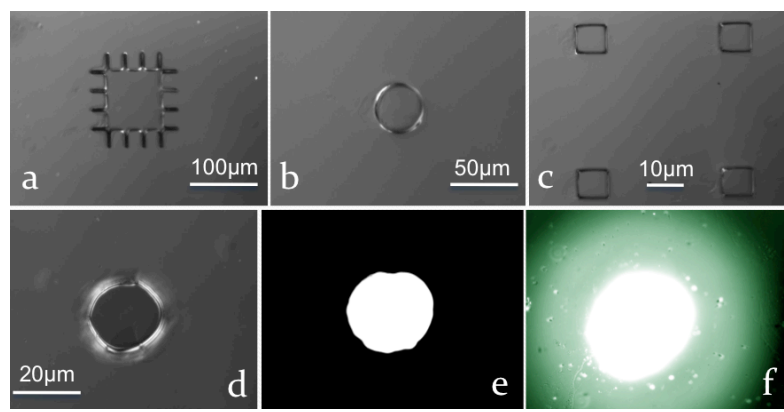

Fig. 4. (a)-(c) Microfabricated hydrogels of different shapes and sizes. (d) Phase-contrast and (e) fluorescent images of a fluorescein doped microgel. (f) Diffusion of fluorescein molecules from the microgel in water.

scraped off the surface of the mold with a clean razor blade. Microgels are then removed from the mold and transferred to the experimental chamber by agitating gels with tweezers.

Hundreds of microgels can be conveniently formed at one time in one mold. Both the PDMS molds and the master posts can be reused at least dozens of times. We fabricate microgels in different shapes and with sizes ranging from 10 to $100 \mu \mathrm{m}$ (see Figure 4a-c). In order demonstrate the ability to deliver chemicals with microgels, we visualize the diffusion profile around the gel using fluorescence. Microgels are doped with fluorescein by mixing the molten agarose solution with $0.1 \%$ fluorescein powder thoroughly before filling the PDMS molds. Figure 4d displays phase contrast images of one of the fabricated microgels. With the addition of fluid, fluorescein molecules start to diffuse and fluorescent microscopy is used to visualize the diffusion (Figure $4 \mathrm{e}$ f). Figure $4 \mathrm{f}$ shows a snapshot of the concentration profile forming around the microgel after one minute.

\section{RESUlts}

In all experiments, an in-plane constant field of $3 \mathrm{mT}$ and a sinusoidal out-of-plane field with amplitude of $1 \mathrm{mT}$ at $30 \mathrm{~Hz}$ is applied to actuate microtransporters that have a characteristic length of $30 \mu \mathrm{m}$. Supporting online video shows the microtransporter performing the tasks presented in this section.

\section{A. Automated transport of Latex Microbeads}

A demonstration of automated transport of a latex microbead is performed using the pose estimate from the tracking algorithm (see Figure 5). The tracking algorithm identifies the position and orientation of the robot as well as a target microbead. A two-step trajectory is planned with movement confined along the cartesian axes. The robot is first controlled to move along the vertical axis at a speed of $6 \mu \mathrm{m} / \mathrm{s}$ until aligned with the microbead. Once aligned horizontally with the microbead, the robot is reoriented for engagement. The bead moves approximately $40 \mu \mathrm{m}$ in the direction of motion of the robot before contact, as would

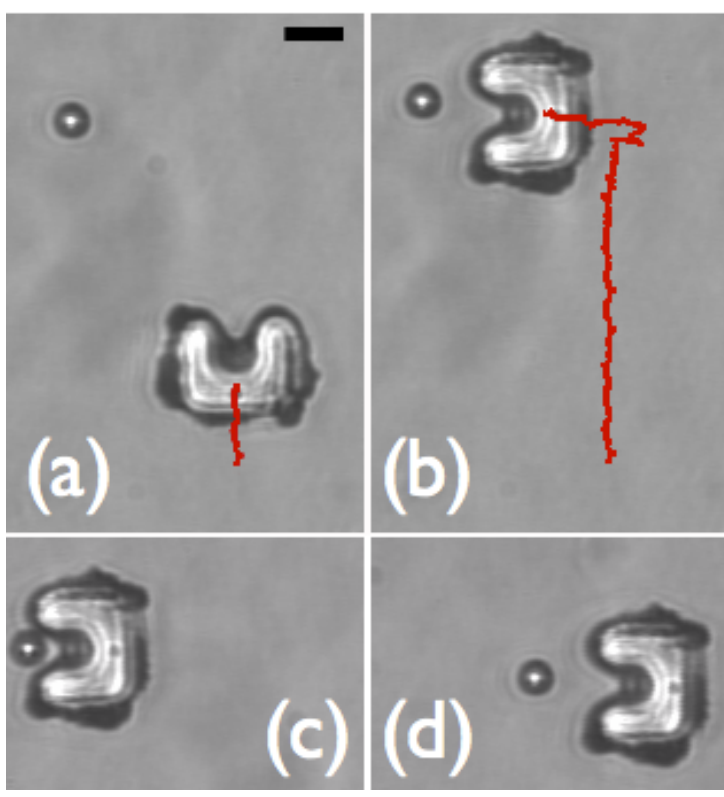

Fig. 5. Automated transport of a $10 \mu \mathrm{m}$ latex microbead. (a) The position of target bead and the microtransporter is detected and used to plan a twostep trajectory. (b) The transporter successfully follows the pre-planned path and engages the target. (c) When the transporter approaches the target, noncontact manipulation is observed. The target bead moves slower than the transport until the transporter comes into contact. (d) The bead is released by moving the transporter back in the same orientation. Again, target moves with the transporter for a while due to fluid coupling. Scale bar is $20 \mu \mathrm{m}$.

be expected due to strong fluid coupling. It should be noted that the exact time of contact is difficult to estimate optically because of the refraction of light by the bead and robot. The bead is released by reversing the direction of motion of the robot while keeping the orientation constant. Again, the bead initially follows the motion of the robot before coming to rest at a position approximately $40 \mu \mathrm{m}$ from its location at the moment of robot reversal.

\section{B. Transport of Agarose Microbeads}

The size and shape of the target object determine the drag coefficient, and change the overall velocity of transport. To characterize the effect of size and estimate the force applied by our microtransporters, we move several different agarose microbeads with sizes ranging from $10 \mu \mathrm{m}$ to $120 \mu \mathrm{m}$ (see Figure 6). We are able to transport beads four times larger and three times smaller in characteristic length than the size of the transporter which shows the scalability of our approach.

The viscous drag force of the fluid acting on a microbead at low Reynolds number regime is given by,

$$
F_{d r a g}=6 \pi \mu R V
$$

where $\mu$ is the viscosity of the fluid, $R$ is the radius and $V$ is the velocity of the microbead. As the size of the transported bead increases, the drag force acting on the bead becomes the 


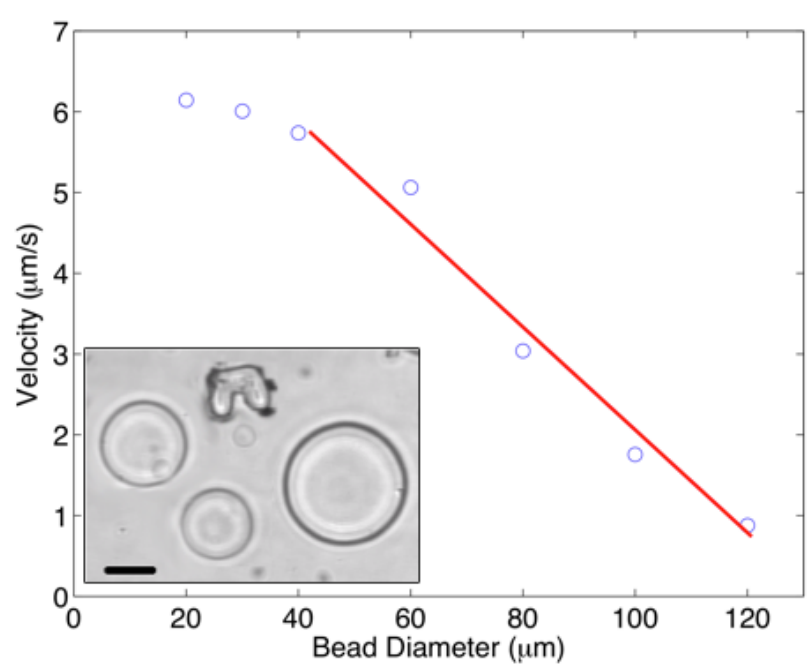

Fig. 6. The velocity of the transporter plus microbead as a function of the size of the microbead. Agarose microbeads in different size and the microrobot are shown in the inset figure. Scale bar is $30 \mu \mathrm{m}$

dominant viscous force for the transporter/microbead pair as the transporter is shadowed by the bead. For beads larger than the size of the transporter $(30 \mu \mathrm{m})$ the velocity is expected to decrease linearly with increasing bead size. Experimental results showed that this is indeed the case (Figure 6). From equation (2), the force applied by the transporter can be estimated. Microbeads with a radius of $60 \mu \mathrm{m}$ are moved with an average velocity of $1 \mu \mathrm{m} / \mathrm{s}$ which gives a force around $1 \mathrm{pN}$ in water at room temperature. We are capable of applying forces in the order of pico Newtons which makes our system safe for manipulation tasks. Mammalian cells can be transported at this force rating without causing any structural damage.

\section{Manipulation of Hippocampal Neurons}

We perform two different experiments with rat hippocampal neurons. In the first experiment, we demonstrate that cells can be transported and released with micron precision. Positioning cells in open and closed microchannels is an important step toward studying cell-cell communication and cell differentiation. In the second experiment, we show the feasibility of delivering drugs to immobilized cells by placing microgels close to neurons patterned on glass slides.

Neuron-enriched primary rat hippocampal cultures are plated at 100,000 per $\mathrm{ml}$ in Neurobasal medium (Invitrogen) with B-27 supplement (Sigma) on poly-L-lysine coated 12$\mathrm{mm}$ round coverslips [17]. Upon attachment to the substrate, a continuous lamella extends around the cell body. This is followed by the emergence of axons. These axons extends for many hundreds of micrometers. After two days, the dendrites begin to grow, and with time the dendrites become more highly branched [18]. After a week, an extensive, intertwined network of axons and dendrites is observed (see Figure 7a).
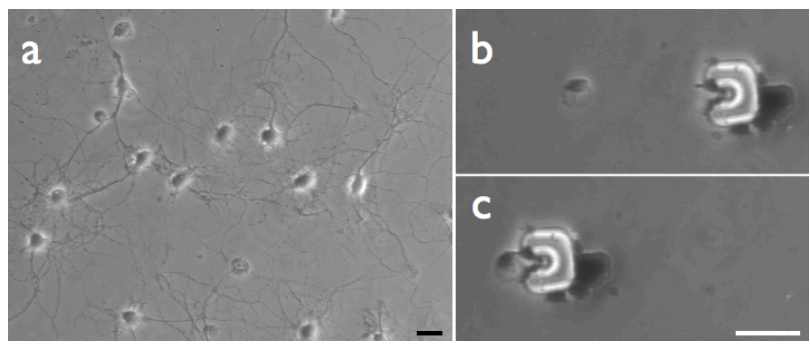

Fig. 7. (a) Phase-contrast images of rat hippocampal neurons. After 10 days in culture, an extensive, intertwined network of neurons develops on glass slides. (b) Transport of trypsinized neurons. A cell is detected and targeted for manipulation. (c) The target is engaged and transported. Scale bars are $25 \mu \mathrm{m}$

1) Transport of cells: We detach cultured neurons from the surface by trypsinizing them in a solution (CMF-HBSS containing $0.5 \mathrm{mM}$ EDTA and $0.05 \%$ trypsin) for $10 \mathrm{~min}$ at room temperature. Trypsin cleaves axons and dendrites and harvested cells change their morphology by taking a ball shape. Their dimensions vary from $10 \mu \mathrm{m}$ to $30 \mu \mathrm{m}$. Cells are transferred onto another cover slip using a micropipette and microtransporters are released into the same fluid.

A microtransporter/target cell pair is selected and a path is planned for the manipulation task (Figure 7b). When the transporter is in close proximity, the cell starts to move due to fluidic effects (Figure 7c). We successfully release the target cell by moving the transporter in the opposite direction without changing its orientation. Adhesion between cells and transporters is observed, but this does not prevent release due to the shape of the robot and surface properties of trypsinized neurons.

2) Delivering microgels to patterned cells: Bioassays using cultured cells for drug screening have focused on the activity of individual cells as well as the function of cell-cell networks in interconnected systems. These are both important measures for drug analysis [19]. A bioassay based on cellular networks would benefit from the techniques of precise patterning of cells and local dosing to the cellular patterns.

Here, a non-invasive technique for delivering small doses of chemicals to a specific area of the patterned rat hippocampal neurons is introduced by using microgels and microtransporters together. Unlike microfluidic solutions, we do not disturb the local chemical environment. Only the individual target cell is manipulated by the presence of gels and robots. Microfabricated $8 \mu \mathrm{m}$ circular microgels and microtransporters are released on 10-day old cultured neurons. Microgels are successfully transported, positioned and released on predetermined spots (Figure 8).

\section{DISCUSSION}

The effect of fluid, physical forces and the concentration of chemicals on cell behavior must be taken into account when designing microenvironments [20]. Our approach is 


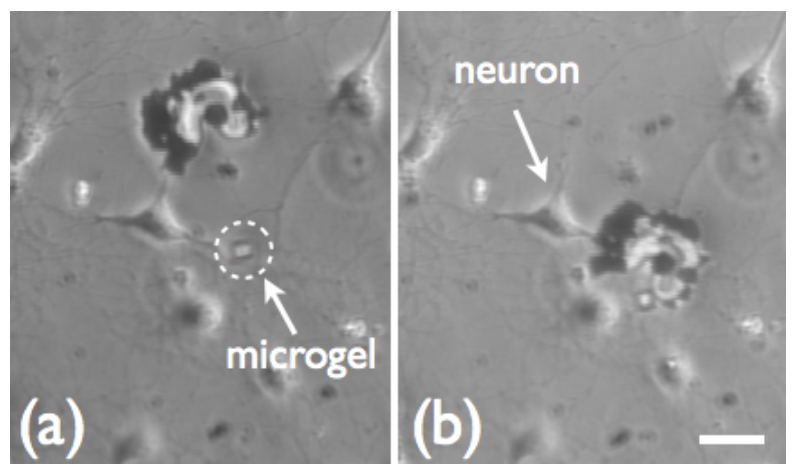

Fig. 8. Delivery of microgels to the hippocampal cultures. (a-b) A microgel is transported from its initial position to its target location. The scale bar is $30 \mu \mathrm{m}$.

non-invasive and requires minimal fluidic disturbance to accomplish manipulation tasks. By integrating microfabricated microgels with magnetic microtransporters, we have shown the feasibility of delivering chemicals locally and engineering more in vivo-like microenvironments in vitro. The effect of multiple growth factors and therapeutic agents on cells can be analyzed by combining mechanical and chemical manipulation. In addition, the shape of the robot is optimized for transport tasks. However, for performing other tasks such as separation, injection, dissection of living cells and applying forces to cultured neurons and fibroblasts, we would like to employ robots with different shapes.

The diffusion kinetics of the delivered chemical needs to be tuned. Drugs should be delivered at pre-determined times and with specific doses. A new technique to develop macroporous ferrogels responsive to magnetic fields has been recently demonstrated [21]. Our future work will address the integration of such active scaffolds with our system for ondemand drug delivery and characterization of the temporal and spatial dynamics of diffusion of chemicals from these smart microgels.

\section{CONCLUSION}

We describe the construction and operation of micronsized, biocompatible ferromagnetic microtransporters driven by external magnetic fields. Our five-coiled, compact actuation system is designed for rapid integration with existing microscopes. We use a real-time visual tracking algorithm for tracking transporters and target objects. This information is used to implement fully automated manipulation of latex microbeads. We also demonstrate the transport of rat hippocampal neurons and fabricated microgels with teleoperation. Microgels are positioned at target locations on cell-patterned surfaces as a first step for delivering drugs to cultured neurons.

\section{REFERENCES}

[1] J. P. Desai, A. Pillarisetti, and A. D. Brooks, "Engineering approaches to biomanipulation," Annual Review of Biomedical Engineering, vol. 9, pp. 35-53, 2007.

[2] S. Martel, C. Tremblay, S. Ngakeng, and G. Langlois, "Controlled manipulation and actuation of micro-objects with magnetotactic bacteria," Applied Physics Letters, vol. 89, 2006

[3] B. Behkam and M. Sitti, "Bacterial flagella-based propulsion and on/off motion control of microscale objects," Applied Physics Letters, vol. 90, 2007.

[4] E. Steager, C.-B. Kim, C. Naik, J. Patel, S. Bith, L. Reber, and M. J. Kim, "Control of microfabricated structures powered by flagellated bacteria using phototaxis," Applied Physics Letters, vol. 90, 2007.

[5] E. B. Steager, M. S. Sakar, D. H. Kim, V. Kumar, G. J. Pappas, and M. J. Kim, "Electrokinetic and optical control of bacterial microrobots," Journal of Micromechanics and Microengineering, vol. 21, 2011.

[6] L. Zhang, J. J. Abbott, L. Dong, B. E. Kratochvil, D. Bell, and B. J. Nelson, "Artificial bacterial flagella: Fabrication and magnetic control," Applied Physics Letters, vol. 94, p. 064107, 2009.

[7] A. Ghosh and P. Fischer, "Controlled propulsion of artificial magnetic nanostructured propellers," Nano Letters, vol. 9, pp. 2243-2245, 2009.

[8] D. R. Frutiger, K. Vollmers, B. E. Kratochvil, and B. J. Nelson, "Small, fast, and under control: wireless resonant magnetic microagents," International Journal of Robotics Research, vol. 29, pp. 613 636, 2010.

[9] M. S. Sakar, E. B. Steager, D. H. Kim, M. J. Kim, G. J. Pappas, and V. Kumar, "Single cell manipulation using ferromagnetic composite microtransporters," Applied Physics Letters, vol. 96, p. 043705, 2010.

[10] T. G. Leong, C. L. Randall, B. R. Benson, N. Bassik, G. M. Stern, and D. H. Gracias, "Thetherless thermobiochemically actuated microgrippers," Proceedings of the National Academy of Sciences, vol. 106, no. 3, pp. 703-708, 2009.

[11] S. Floyd, C. Pawashe, and M. Sitti, "Two-dimensional contact and noncontact micromanipulation in liquid using an untethered mobile magnetic microrobot," IEEE Transactions on Robotics, vol. 25, pp. 1332-1342, 2009.

[12] C. Pawashe, S. Floyd, and M. Sitti, "Multiple magnetic microrobot control using electrostatic anchoring," Applied Physics Letters, vol. 94, p. 164108, 2009.

[13] V. Linder, B. D. Gates, D. Ryan, B. A. Parviz, and G. M. Whitesides, "Water-soluble sacrificial layers for surface micromachining," Small, vol. 7, pp. 730-736, 2005.

[14] M. S. Sakar, E. B. Steager, D. H. Kim, A. A. Julius, M. J. Kim, V. Kumar, and G. J. Pappas, "Biosensing and actuation for microbiorobots," in IEEE International Conference on Robotics and Automation, Anchorage, AL, 2010, pp. 3141-3146.

[15] C. Pawashe, S. Floyd, and M. Sitti, "Modeling and experimental characterization of an untethered magnetic micro-robot," International Journal of Robotics Research, vol. 28, pp. 1077-1094, 2009.

[16] A. P. McGuigan, D. A. Bruzewicz, A. Glavan, M. Butte, and G. M. Whitesides, "Cell encapsulation in sub-mm sized gel modules using replica molding," PLOS one, vol. 3, p. e2258, 2008.

[17] L. E. Barrett, J.-Y. Sul, H. Takano, E. J. V. Bockstaele, and P. G. Haydon, "Region-directed phototransfection reveals the functional significance of a dentrically synthesized transcription factor," Nature Methods, vol. 3, pp. 455-460, 2006.

[18] S. Kaech and G. Banker, "Culturing hippocampal neurons," Nature Protocols, vol. 1, pp. 2406-2415, 2006.

[19] H. Kaji, M. Nishizawa, and T. Matsue, "Localized chemical stimulation to micropatterned cells using multiple laminar flows," Lab on a Chip, vol. 3, pp. 208-211, 2003.

[20] G. M. Walker, H. C. Zeringue, and D. J. Beebe, "Microenvironment design considerations for cellular scale studies," Lab on a Chip, vol. 4, pp. 91-97, 2004.

[21] X. Zhao, J. Kim, C. A. Cezar, N. Huebsch, K. Lee, K. Bouhadir, and D. J. Mooney, "Active scaffolds for on-demand drug and cell delivery," Proceedings of the National Academy of Sciences, vol. 108, pp. 67-72, 2011. 